\title{
tic\&société
}

Vol. 6, $\mathrm{N}^{\circ} 1$ | Second semestre 2012

Mutations du journalisme : nouveaux dispositifs, nouvelles pratiques

\section{Presse régionale et multisupports}

les petits pas de géants

\section{Denis RUELLAN et Joël LANGONNE}

\section{OpenEdition}

Journals

Édition électronique

URL : http://journals.openedition.org/ticetsociete/1203

DOI : 10.4000/ticetsociete. 1203

Éditeur

Association ARTIC

Référence électronique

Denis RUELLAN et Joël LANGONNE, «Presse régionale et multisupports », tic\&société [En ligne], Vol. 6, $N^{\circ} 1$ | Second semestre 2012, mis en ligne le 28 novembre 2012, consulté le 03 mai 2019. URL : http:// journals.openedition.org/ticetsociete/1203; DOI : 10.4000/ticetsociete.1203 


\title{
Presse régionale et multisupports : les petits pas de géants
}

\author{
Denis RUELLAN \\ Professeur des universités \\ Université de Rennes 1 \\ UMR 6051 CRAPE \\ denis.ruellan@gmail.com \\ JoëI LANGONNE \\ Doctorant \\ Université de Rennes 1 \\ UMR 6051 CRAPE \\ joel.langonne@gmail.com
}

Denis RUELLAN, professeur des universités, est membre du laboratoire Centre de recherche sur l'action publique en Europe (CRAPE). Ses recherches portent principalement sur la construction de l'identité professionnelle, il adopte une perspective socio-anthropologique qui le conduit à explorer les phases de l'histoire du journalisme et des journalistes pour éclairer les questions contemporaines. II a publié récemment Nous, journalistes. Déontologie et identité, PUG, 2011, une étude sur la place du discours moral dans l'élaboration des relations entre pairs, avec les sources, avec les employeurs et avec les publics.

Joël LANGONNE, doctorant en Sciences de I'Information et de la Communication à l'université Rennes 1, est membre du laboratoire Centre de recherche sur l'action publique en Europe (CRAPE). Ancien journaliste, ses travaux de recherche envisagent le journal comme une œuvre collective, travaillée par un ensemble disparate d'acteurs humains et non-humains qui agissent tout au long du continuum de fabrication de l'information. II s'agit d'observer plus particulièrement des zones un peu floues de cette dynamique, des espaces entre le dessein du journal et son dessin effectif. Ces interstices sont autant de possibilités de médiations pour les acteurs qui s'y déploient. Ainsi, l'objet journal est nécessairement plastique. Selon les époques, et de façon non-linéaire, il est plus ou moins chargé des présences au monde de tous ceux qui participent à son élaboration. 


\title{
Presse régionale et multisupports : les petits pas de géants
}

\begin{abstract}
Résumé
Cet article se propose de revenir sur la trajectoire suivie par un quotidien régional dans la diversification de ses activités et la mise à contribution de ses équipes pour réaliser un journalisme multisupports. Elle soutient l'hypothèse que l'objectif de ce processus est moins dans la recherche d'une transformation des activités traditionnelles que dans la construction d'un discours de modernité et dans l'acculturation à des activités nouvelles qui peuvent être développées parallèlement au journal papier.
\end{abstract}

Mots-clés : multisupports, presse, journalisme

\section{Abstract}

This article retraces the path followed by a regional daily in France in diversifying its activities and enlisting its workers in the task of mastering a multichannel journalism. It supports the hypothesis that the objective of the process is less the transformation of the traditional activities of that enterprise than the construction of a discourse of modernity and a process of acculturation of its workers to new activities that can now be developped in parallel to the production of the newspaper.

Keywords: multichannel, press, journalism, local

\section{Resumen}

Este artículo pretende estudiar la trayectoria de un periódico regional francés en la diversificación de sus actividades, así como la contribución de sus equipos para llevar a cabo un periodismo multisoporte. En la base de dicha trayectoria se sitúa la hipótesis de que el objetivo se sitúa no tanto en la búsqueda de una transformación de las actividades tradicionales como en la construcción de un discurso de modernidad y en la aculturación de nuevas actividades que pueden desarrollarse en paralelo al periódico en soporte papel. 
Presse régionale et multisupports :

les petits pas de géants

Palabras clave: multisoporte, prensa, periodismo

Cet article se propose de réinterroger la notion de journalisme multisupports, très présente dans le monde des médias ces dernières années, et qui a pu être exprimée sous des vocables assez proches de multimédias ou de convergence. Un journaliste essayiste prédisait il $\mathrm{y}$ a près de trente ans à propos de son propre métier : "Le journaliste de demain sera - selon un terme affreux mais expressif - multimédia, c'est à dire qu'il passera, parce qu'il en maîtrisera toutes les techniques, de ce que nous appelons encore "presse imprimée" aux diverses formes de celles qui sont ou seront transmises par les ondes et les écrans. Le processus de la communication tendra de plus en plus vers l'unité, mais en se diversifiant toujours davantage. " (Voyenne, 1985). II observait alors que l'adoption de systèmes informatisés à l'Agence France-Presse allait se généraliser à l'imprimé, que l'écran de l'ordinateur allait devenir le centre de la production des nouvelles et conduire le journalisme à ce que nous désignons multisupports, une diversification dans l'intégration.

Le journalisme multisupports est lié à la numérisation de la chaîne de fabrication des journaux. Cette numérisation, totale à la fin des années 1990, a ouvert la voie à la circulation de contenus identiques ou enrichis sur plusieurs supports (papier, internet, télévision, mobiles, etc.). Cela peut impliquer la mise en place d'organisations nouvelles du travail, et une modification de la compétence et des fonctions des journalistes. Dans ce contexte, ils doivent produire à la fois du texte, du son et de l'image, ils doivent ainsi pratiquer un journalisme sur de multiples supports. Le terme s'inscrit aussi dans un processus plus large qui concerne la diversification des activités des firmes de médias, quand celles-ci tendraient elles aussi à devenir multisupports, en rationalisant les process et en valorisant les produits sur plusieurs activités.

Les deux mouvements sont en fait liés. Les entreprises de presse sont attentives à ces possibilités de transformation qui constituent à cette époque des pistes sérieuses pour faire face à la crise. Mais les discours qu'elles portent sur ces évolutions restent, pour beaucoup, des projections sur l'avenir, des anticipations sur une réalité encore peu nette ; ces discours agitent l'imaginaire d'un journaliste Shiva ou backpack, capable de mobiliser toute la batterie des outils du reporter: stylo, caméra, micro, téléphone, etc. Côté académique, des recherches ont très vite exposé les mutations de la filière industrielle vers le multisupports (Quinn, 2004 ; Forte Duhe, Mortimer et Chow, 2004 ; Jenkins, 2004 ; Quinn et Quinn-Allan, 2005 ; Domingo et al, 2007 ; Bouquillon, 2008), elles ont aussi tenté de comprendre comment ces transformations pouvaient conduire les journalistes à devenir des acteurs d'une organisation entrepreneuriale de ce type (Garcia et Bienvenido, 2002; Singer, 2004; 


\section{Denis RUELLAN et Joël LANGONNE}

Cabrolié, 2009 et 2010). Ces travaux convergent pour indiquer que le passage au multisupports, quand il a lieu, est un processus qui n'est pas linéaire, qui n'est pas uniforme (Deuze, 2005 ; Domingo et al., 2007). Les transformations qui s'opèrent sont à comprendre dans des continuums toujours spécifiques (Gestin et al., 2009), ce que les entreprises de presse ont fini par reconnaître elles-mêmes, après avoir un temps promu l'idée qu'un modèle de rationalisation de la production médiatique aurait raison de la crise économique qui affecte lourdement le sous-secteur de la presse. Ainsi l'IFRA (INCA-FIEJ Research Association, aujourd'hui baptisée WAN-IFRA), l'organisation mondiale qui réunit la plupart des groupes spécialisés dans la fabrication de journaux, finit par soutenir que la convergence ne se décrète pas et s'applique encore moins selon un modèle. "II n'existe pas une seule manière d'organiser la salle de rédaction multimédia. Néanmoins (...) un point commun réunit ces expériences : il ne faut pas figer les nouvelles organisations, mais rester dans un processus d'apprentissage et d'adaptation ${ }^{\prime}$ ».

C'est donc cette contextualité des processus de restructuration que nous interrogeons ici, en prenant appui sur l'exemple en France d'un titre de presse quotidienne régionale $\left(\mathrm{PQR}^{2}\right)$, Le Télégramme. Ce quotidien a diffusé en 2011 un peu plus de 212000 exemplaires sur trois départements pour dix-huit éditions, en Bretagne. II emploie 550 personnes, dont 220 journalistes et s'attache les services de 600 correspondants locaux de presse (CLP3). II réalise, depuis plusieurs années, la meilleure progression de diffusion de l'ensemble des quotidiens régionaux. II s'agit du titre phare d'un groupe de presse qui s'est beaucoup impliqué, depuis le milieu des années 1990, dans une stratégie de développement par la diversification. Ainsi, si le journal représentait la quasi totalité du chiffre d'affaires (CA) de l'entreprise il y a quinze ans, il ne pesait plus en 2010 que pour les deux tiers des 135 millions d'euros de CA réalisés par le groupe. C'est le résultat d'une politique de diversification qui a conduit à la création ou à l'achat de filiales spécialisées dans la communication, les petites annonces, la gestion d'événements, les services sur internet. Ce tournant, s'il est à l'évidence lié à une évolution profonde de la structure

\footnotetext{
${ }^{1}$ VESELING B., 2009, «Trois rédactions font un bilan de leur réorganisation », IFRA Magazine, juillet, pp. 12-15.

${ }^{2}$ La PQR, en France, se caractérise par des journaux dont la couverture est limitée à une région ou plusieurs régions. Son contenu est axé sur l'information de proximité. Elle domine le paysage de la presse en France, où elle représente plus de la moitié des exemplaires de presse quotidienne, avec 53 titres, pour plus de 18 millions de lecteurs, contre 16 titres et 8 millions de lecteurs pour la presse quotidienne nationale (PQN).

${ }_{3}^{3}$ Les CLP sont des collaborateurs des journaux de PQR, présents sur l'ensemble du territoire de diffusion des titres. Le CLP n'est pas un journaliste, il est un travailleur indépendant, rémunéré à l'acte, et dont la production est soumise à appréciation de journalistes professionnels avant parution.
} 
Presse régionale et multisupports :

les petits pas de géants

économique dans laquelle évolue la presse écrite, tient également à l'arrivée aux commandes de l'entreprise d'une nouvelle génération. Comme la société anonyme (SA) créée à la Libération pour publier le quotidien régional, la société qui chapeaute le groupe, fondée en 2007, est détenue majoritairement par une seule famille. Cette dernière a toujours veillé à autofinancer ses investissements, même lorsqu'il s'agissait, comme en 1996 et 2001 de s'équiper de deux nouvelles rotatives d'un coût unitaire de l'ordre de huit millions d'euros. Elle est aussi parvenue à tirer profit de la position particulière du Télégramme: un journal dont le territoire de diffusion semble a priori inconfortablement positionné entre l'océan à l'ouest et le géant Ouest France à l'est, premier titre de PQR diffusant à plus de 760.000 exemplaires. C'est précisément à partir de ces singularités que les dirigeants sont parvenus à conférer une identité particulière au titre et, par extension, à l'entreprise. Le Télégramme serait ainsi un groupe de presse indépendant, assiégé à la pointe de l'Europe par des mastodontes de la communication. Il se doit donc d'être innovant, dynamique, réactif etc., question de survie. Ce type de discours aura, on va le voir, une importance particulière dans le processus qui nous intéresse.

Nous allons montrer ici que, malgré l'intérêt précoce du groupe pour un passage au multisupports, celui-ci ne s'est pas réalisé, pas autant que projeté pour le moins, et tenter d'expliquer cet état de fait. Notre hypothèse est que l'objectif premier n'était pas forcément qu'il se réalise en tant que tel. D'autres buts étaient aussi poursuivis, et ont été réalisés. Notre recherche se fonde sur une observation dans la durée, alimentée d'entretiens menés sur trois périodes (2008, 2010, 2012), avec différents acteurs du groupe: des journalistes généralistes, des journalistes spécialisés, des cadres de l'entreprise, des responsables de la rédaction, des techniciens et des représentants syndicaux. Une trentaine d'entretiens en tout. Ce travail vient en écho à des recherches à peu près équivalentes menées dans quatre autres groupes de presse et communication: Bolloré, L’Équipe, Le Progrès en France, Québecor au Canada ${ }^{4}$ à la même époque.

\footnotetext{
${ }^{4}$ Ce programme a été conduit par I'UMR CRAPE, avec le soutien du GIS M@rsouin. Gestin et al., 2009. II a impliqué Christophe GIMBERT, Maître de conférences, CRAPE (UMR CNRS 6051), Université de Rennes 1; Joël LANGONNE, doctorant, CRAPE (UMR CNRS 6051), Université de Rennes1; Florence LE CAM, Maître de conférences, CRAPE (UMR CNRS 6051) \& ReSIC Université Libre de Bruxelles (Belgique) ; Magali PRODHOMME, Maître de conférences, CRAPE (UMR CNRS 6051), Université catholique de l'Ouest, Angers (France) ; Yvon ROCHARD, Maître de conférences associé, Université de Rennes 1 ; Hélène ROMEYER, Maître de conférences, CRAPE (UMR CNRS 6051) \& GRESEC (EA MRT 608), Université de Rennes 1; Denis RUELLAN, Professeur des universités, CRAPE (UMR CNRS 6051), Université de Rennes 1.
} 


\section{S'émanciper du print : la mise en œuvre du journalisme multisupports au Télégramme}

Commençons par signaler que pendant longtemps, dans l'entreprise de presse étudiée, le développement d'une stratégie multisupports n'a pas vraiment fait l'objet d'un discours unifié. Plusieurs cadres de la rédaction circulent dans les milieux nationaux et internationaux des entreprises de presse, s'imprègnent des expériences en cours, mais la perspective d'un passage au multisupports demeure à un niveau informel d'expression. Ce n'est qu'en 2006 que le discours des responsables du quotidien se précise. Ils souhaitent passer à une logique qui permet de s'émanciper du contenu papier. En fait, ils veulent réutiliser ce contenu tout en s'affranchissant des limites géographiques, thématiques et de forme éditoriale du journal. Pour la direction, le print est parvenu aux limites de son développement. Les investissements dans l'internet paraissent les plus intéressants pour l'avenir. "On était limité à l'écrit, sur un territoire limité, on se retrouve entreprise mondiale sur des supports multiples ». Trois ans plus tard, au tout début 2009, ils lancent un vaste plan de développement multisupports, après la signature d'un accord d'entreprise. Les signataires disent se fixer pour objectif «l'enrichissement de l'offre rédactionnelle du journal par la production de nouveaux contenus numériques ».

Pour y parvenir, les signataires décident qu'il faut former les journalistes, leur donner de nouveaux outils pour travailler, et nommer des professionnels motivés pour initier et pérenniser les nouvelles façons de capter et de transmettre l'information. Si on revient sur ce plan de développement multisupports et sur ce qu'il a effectivement impliqué, on peut aujourd'hui faire quelques constats, et avoir autant de raisons de relativiser, en quelque sorte, les effets et la portée du passage au multisupports :

\subsection{Une rédaction formée}

Un des points clés de l'accord stipulait que les journalistes devaient désormais être en mesure de produire et de transmettre des images et du son destinés au site internet. Ce qui a beaucoup marqué les journalistes, c'est l'effort considérable fait par leur entreprise pour les former à ces nouvelles tâches. Entre 2009 et 2010, 220 journalistes - l'ensemble de la rédaction - ont suivi un module de tronc commun sur deux jours, une formation de "culture générale multimédia " visant à apporter un "socle commun" de connaissance sur les outils numériques et une initiation à la vidéo. Une cinquantaine de journalistes a bénéficié d'une formation de quatre jours à la prise de vue vidéo, une petite 
Presse régionale et multisupports :

les petits pas de géants

dizaine a été formée au montage vidéo (quatre jours), une vingtaine a suivi un module de deux journées consacré à l'écriture sur internet. Ce volet formation du plan de développement multisupports, qui a constitué le gros des investissements de l'entreprise, a été mené sous la houlette de I'IFRA. Le choix de ce prestataire est, on le verra, loin d'être anodin. Ces formations ont été unanimement appréciées, pour la qualité des formateurs et pour leur adaptation aux attentes. Pourtant, elles n'ont pas occasionné d'effets notables, hormis à très court terme, sur l'implication des journalistes dans la production de vidéos.

\subsection{De nouveaux outils}

On a dit que ces journalistes formés devaient pouvoir disposer de nouveaux outils. À ce sujet, et c'est notre deuxième constat, il faut noter la modestie des investissements mis en place dans le cadre du plan : 35 caméscopes ont été achetés et répartis dans les services et les agences (deux dans les plus grosses agences, une dans les petites) ; ils sont conçus pour la captation d'images d'une qualité suffisante pour une édition ultérieure. 135 journalistes ont été équipés de téléphones dotés d'un enregistreur vidéo pouvant capter de courtes séquences, ainsi que des photographies et du son. On peut aussi ajouter la mise en place d'une petite régie d'édition.

Passé un relatif enthousiasme au début, les journalistes localiers ne produisent pratiquement pas de vidéo. Les rédactions peinent à intégrer une forme de «penser web », qui pourrait permettre d'alimenter le site du journal en images animées, dans une organisation quotidienne déjà serrée. En fait, les caméscopes ne sortent pas des rédactions. De même, les professionnels n'utilisent que très peu les fonctions vidéo ou prise de son de leur smartphone. Le modèle dont ils ont été dotés en 2009 était déjà obsolète, et les rares journalistes qui se sont tout de même «coltinés » à l'objet ont rapidement mis en évidence des difficultés d'utilisation, en particulier dans la qualité de la couverture, ce qui rendait difficile la transmission de données via cet outil.

\subsection{Des journalistes "référents multimédia »}

Dernier constat, lié aux deux précédents: le plan multisupports n'a pas occasionné de réorganisation significative des processus de travail. Au niveau des intentions, les journalistes étaient formés aux nouvelles pratiques, et disposaient d'outils pour les mettre en œuvre. Pour les aider dans ces nouvelles tâches, la direction a recruté trois journalistes, baptisés « référents multimédia », qui devaient être l'un des moteurs du basculement de toute la rédaction dans le multisupports. Dans le plan, ces référents étaient tout particulièrement dédiés à 
l'appui des journalistes localiers. Ils devaient les inciter à pourvoir aux besoins du site, et notamment aux besoins en vidéos.

Mais d'emblée, leur fonction a flotté. En fait, les référents ne sont jamais parvenus à pousser leurs collègues des rédactions locales 5 à entrer dans une pratique multisupports, par manque de temps, de motivation aussi. Du coup, un peu par la force des choses, les référents se sont mis à produire toute la vidéo à la place des journalistes localiers, et sont devenus eux-mêmes des producteurs d'images. Signe de leur inadéquation aux besoins, lors de la renégociation de l'accord en 2011, les trois référents multimédia n'ont pas été reconduits dans ce titre, et ont rejoint d'autres postes.

Ainsi, hormis la production de vidéos, qui demeure à la marge comme on vient de voir, on peut dire que le multisupports est entré dans le quotidien des journalistes essentiellement par la petite porte technique. Dans les faits, la majeure partie des journalistes de terrain se cantonne à effectuer deux autres tâches stipulées dans l'accord: ils doivent tous ajouter des mots-clés à chaque article ou chaque photo publiée, pour faciliter le référencement sur les moteurs de recherche. Lorsque l'actualité le commande, ils doivent aussi rédiger des brèves qui seront mises en ligne en temps réel. D'une certaine façon, il s'agit là de techniques de communication qui relèvent plus de l'opérateur technique que du journaliste multisupports.

\subsection{Les oubliés du plan multisupports}

Nous venons de tracer à grands traits les principaux axes du plan multisupports, en soulignant les limites dans sa mise en œuvre. Une autre limite de ce plan est ce qu'il ne contient pas, et qui concerne - le cas échéant, qui ne concerne pas - deux acteurs du monde de la PQR : les journalistes secrétaires de rédaction (SR) et les correspondants locaux de presse (CLP).

La plupart de nos interlocuteurs ont souligné, lors de notre enquête, l'une des forces du Télégramme: son réseau de CLP, qui lui permet de disposer d'un maillage territorial performant sur l'ensemble de son aire de diffusion. Dans l'organisation du travail, le référent du CLP au sein du journal est le journaliste SR responsable de l'édition pour laquelle le correspondant produit des articles. Disons, pour simplifier, que le CLP transmet les articles qu'il a écrit à un SR, qui

\footnotetext{
${ }^{5}$ Les titres de PQR en France peuvent compter, en plus de leur(s) rédaction(s) principale(s), des rédactions plus modestes, disséminées sur leur territoire de diffusion. Ces rédactions "locales" n'abritent qu'un ou deux journalistes, dits "localiers". Le Télégramme, parmi ses 21 rédactions, compte six rédactions importantes et quinze locales.
} 
Presse régionale et multisupports :

les petits pas de géants

se charge de les valider et de les mettre en page. CLP et SR sont donc intimement liés dans la chaîne de production de l'information locale.

Lors des négociations de 2008, qui précédèrent la mise en place du plan de développement multisupports, ce lien entre CLP et SR a été discuté, et certains négociateurs ont indiqué qu'il était sans doute possible faire contribuer directement ces deux acteurs à une organisation du travail multisupports. II fut ainsi question de revoir la position éditoriale des SR. II s'agissait par exemple, pour chaque plateau de secrétariat de rédaction du journal ${ }^{6}$, de dédier un $S R$ à la mise en ligne d'une partie de la production des CLP sur le site web du journal.

Ces propositions n'ont rien donné. Au fond, il nous semble qu'elles ne pouvaient pas aboutir sous cette forme. Le discours qui présente les CLP comme des acteurs incontournables de la PQR - ce qu'ils sont en effet - est complété par un autre, qui, au Télégramme, interroge directement l'utilité des correspondants locaux de presse, qui désavoue même les CLP. Au fond, les correspondants ne donneraient pas satisfaction. II s'agit cette fois de décrire des CLP trop marqués par leurs propres convictions ou affections, pas faciles à employer, rares ou pas assez fiables. II s'agit surtout d'indiquer, en filigrane, que les CLP apportent une information qui pourrait être captée autrement et sans frais via les réseaux numériques. Ces interrogations justifient que la production des correspondants ait été clairement exclue de l'accord de 2008.

II y a sans doute autre chose : inclure la production des CLP dans l'accord aurait nécessité de questionner la place et le rôle des $S R$, leurs interlocuteurs dans le journal. Or, la place et le rôle des secrétaires de rédaction sont, à cette époque, problématiques au Télégramme - ils le sont toujours. Depuis quelques années en effet, tous les journalistes sont encouragés à acquérir la double compétence de SR et de rédacteur, privant ainsi, si l'on peut dire, les SR de leur spécificité. Par ailleurs, et c'est lié, la rédaction en chef évoque la possibilité, pour des rédacteurs qui exercent dans les locales, formés au secrétariat de rédaction, de construire eux-mêmes leurs pages, s'affranchissant ainsi de l'étape du plateau SR.

Ces aspirations de la hiérarchie restent pour le moment sans effet ou presque : au Télégramme, les CLP demeurent indispensables et chacune des pages des éditions reste montée sur l'un des six plateaux de secrétariat de rédaction. De manière pragmatique cependant, mieux valait en 2008 oublier d'embarquer ces acteurs dans le plan multisupports, pour ne pas devoir légitimer, ou relégitimer, les positions de ces deux groupes, pour ne pas

\footnotetext{
${ }^{6}$ Qui en compte six au total.
} 
compromettre un futur - et toujours hypothétique - redéploiement de la place et du rôle des CLP et des SR au sein du journal.

Globalement, ces constats nous permettent de souligner la relative faiblesse des moyens qui auraient dû soutenir un développement rapide des compétences et des activités multisupports des équipes rédactionnelles: les investissements sont modestes, les redéploiements de postes peu efficients et finalement l'accompagnement dans le temps de la politique entamée est limité. Les résultats s'en ressentent: le multisupports est passé au second plan des projets trois ans après son lancement officiel.

\section{Se rassembler pour se diversifier}

$\mathrm{Au}$ vu de ces constats, on pourrait conclure à un échec du plan de développement multisupports. Mais il nous faut garder à l'esprit que les processus de convergence à l'œuvre sont toujours inscrits dans des continuums spécifiques. Cela nous amène à deux hypothèses: d'une part, pour cette entreprise de presse, le thème du multisupports a constitué un discours de réassurance, à l'interne et à l'externe. Ensuite, c'est notre deuxième hypothèse, le programme, pris dans son ensemble depuis six ans, constitue une acculturation graduelle à deux nouveaux domaines : I'Internet et la télévision.

\section{$2.1(R e) d i r e$ " nous ", dans et hors les murs de l'entreprise}

Discours de réassurance à l'externe, d'abord, car l'une des intentions de ce plan était pour l'entreprise de faire la preuve publique de sa capacité d'adaptation aux nouveaux paysages de l'information. Le titre souhaitait faire savoir qu'il était capable de s'inscrire dans le mouvement de diversification des supports et de globalisation des échanges.

Le multisupports porte en lui-même un discours de l'innovation, exprimé par des initiatives concrètes et originales, et qui s'adresse aux lecteurs et clients, et aussi à la communauté professionnelle des médias. On peut par exemple indiquer que l'entreprise s'est assurée les services de l'IFRA pour former ses journalistes aux pratiques multisupports. Le choix de ce prestataire est loin d'être anodin. Dans ces années-là, l'IFRA - qui se dit l'« organisation mondiale de recherche et de services pour l'industrie de la presse " et à ce titre propose des activités de conseil, de formation - apparaît comme l'un des acteurs les plus influents pour la promotion et la mise en place d'une organisation multisupports et intégrée dans les entreprises de presse - la « convergence » est le terme 
Presse régionale et multisupports :

les petits pas de géants

utilisé à cette époque. Faire appel à I'IFRA, bénéficier de ses services (ici des sessions de formation), c'est aussi octroyer à son entreprise et à ce qui s'y passe une visibilité considérable dans le monde des entreprises de presse. Cela participe à entretenir, à renforcer, une image de succès - et aussi de trublion qu'a le groupe Le Télégramme au sein du secteur plutôt conservateur et mal en point de la $P Q R$.

À ce moment précis de la trajectoire de l'entreprise, ce plan s'inscrit aussi dans une dynamique de réassurance à l'interne, car le plan de développement multisupports a constitué un discours rassurant pour soi. Pour bien comprendre cela, il faut procéder à un bref retour en arrière. En 1996, la direction s'engage avec volontarisme et sans concertation dans l'aventure Internet: un portail régional est mis en place, ainsi qu'un site. La totalité du contenu du journal est mis en ligne, à l'exception des petites annonces. Toute l'activité internet est organisée par une société d'investissement. Elle gère le portail régional, des city guides, un site dédié à la voile, et un autre à la musique. Le pôle internet multiplie rapidement les initiatives, travaille sur quantité de projets, embauche... II y a une dissociation presque totale entre les projets print et web. Les deux équipes travaillent en parallèle, sans relation. En 2002 (éclatement de la « bulle internet »), les vents tournent, l'hébergeur met la clé sous la porte et le site est gelé. Cet échec est vécu comme un traumatisme. La direction du groupe voit la confiance des salariés atteinte, dans une entreprise où l'attachement de chacun au projet collectif est un des moteurs de la réussite.

Ainsi, le développement du multisupports de 2009 a-t-il aussi pour objectif de cautériser ces plaies internes, qui se révèlent par un sentiment d'incertitude, voire de défiance à l'égard des choix de la direction concernant internet. L'intention du plan est aussi de restaurer une confiance, en replaçant la rédaction au centre du processus d'innovation. Néanmoins, ce programme de réassurance n'était pas que cela, et c'est là l'autre pan de l'hypothèse : il a aussi permis à l'entreprise de réaliser des expérimentations dont elle a fini par tirer profit par une extension de sa compétence vers l'internet, et vers la télévision.

\subsection{Internet dans le giron rédactionnel}

On vient de l'évoquer, la première aventure internet de l'entreprise, qui externalisait le développement des activités internet, fut un échec. En 2004, quelques années avant le plan de développement multisupports, le dossier est repris, par la rédaction cette fois, sous la houlette de la rédaction en chef. En novembre 2005, un site modernisé est mis en ligne. Il est supervisé par le service de documentation, qui fait partie de la rédaction. Ce service 
documentation se positionne d'ailleurs comme LA composante multimédia interne.

Comme avant, l'essentiel de son contenu est issu du journal papier, encore systématiquement basculé chaque nuit ; quelques enrichissements ont tout de même eu lieu, tels que la mise en place de fils d'actualité (textes et vidéos) issus d'agences et surtout la mise en ligne de dossiers, constitués spécialement par le service de documentation du quotidien. Malgré ces évolutions, la direction éditoriale du site reste floue. Elle semble subordonnée à la mise en place de moyens, qui permettraient une utilisation différente des ressources propres du quotidien, une utilisation adaptée au support. Le plan de développement va permettre cette adaptation.

$\mathrm{Au}$ premier trimestre 2009 , le site est remanié. II est cette fois sous la houlette d'un véritable service multimédia, doté de onze personnes, dont les journalistes de la documentation. Le nouveau site est enrichi de brèves d'actualité, de dossiers spécifiques, d'éléments en prolongement et en complément du print, faits de textes, de photos, de son et de vidéo. II revendique aujourd'hui plus de 5 millions de visites chaque mois ${ }^{7}$, ce qui le place au troisième rang des sites de PQR (derrière ouest-france.fr et ladepeche.fr).

Même si la plupart des journalistes ne produisent peu ou pas de vidéos, le travail des référents multimédia - qui sont devenus les producteurs de vidéo, on l'a dit - est à cette époque largement suffisant pour alimenter le site en images et en sons. Un site qui est donc doté désormais d'une offre assez distincte de celle du papier.

\subsection{De l'information “ comme à la télé »...}

Tout comme le volet internet, le projet de télévision n'est pas né avec le plan de développement multisupports. Le projet s'ancre avant le plan, et se poursuit aujourd'hui, alors que le multisupports est relégué au second plan. En fait, ce plan a permis à l'entreprise de se doter d'une expertise télévisuelle. Elle a pu dire : " on sait faire de la télé ". II a en quelque sorte légitimé un grand projet entrepreneurial : la création d'une chaîne régionale.

Avant celle-ci, le groupe s'était déjà illustré par une innovation remarquée et saluée dans le monde des médias : le JT-Web. Une expérimentation qui a tenu dix-huit mois à partir du printemps 2007. Le JT-Web est un authentique journal

\footnotetext{
${ }^{7}$ Source OJD, pour le mois d'avril 2012.
} 
Presse régionale et multisupports :

les petits pas de géants

télévisuel de 5 minutes environ, diffusé à partir de 17 heures cinq fois par semaine sur le site. Il est réalisé par une équipe resserrée, à cheval entre le service des informations générales (IG) et la documentation. C'est un journaliste reporter d'images (JRI) spécialement embauché pour le JT-Web qui produit les vidéos. Ce JT-Web n'a pas remporté un grand succès (environ 1200 visites par jour, contre 50000 pour le site dans son ensemble, à cette époque). L'insuccès est expliqué par une double inadaptation. D'abord, la forme copiée de la télévision n'est pas faite pour la consommation sur internet, qui privilégie la brièveté et la circulation à travers des contenus variés. Ensuite, le JT-Web comme addition linéaire de plusieurs sujets est un objet inconnu des moteurs de recherche, qui ne référencent que des produits dont le principe est l'unicité thématique ; ainsi, même si un JT-Web contient une séquence susceptible d'une forte circulation (buzz), celle-ci ne sera pas perçue par les automates et donc pas valorisée.

Au printemps 2008, le JT-Web est supprimé, au profit de vidéos autonomes installées dans tous les coins du nouveau site. Pourtant, l'expérience acquise avec le JT-Web est loin d'être un échec. Elle est clairement réinvestie dans la construction de la nouvelle chaîne de télévision régionale, Tébéo. Les journalistes qui ont participé au JT-Web, acculturés par l'expérience, vont rejoindre ce nouveau projet.

\subsection{Au-delà du multisupports}

Dans l'entreprise de presse qui nous intéresse, le multisupports ne s'est pas réalisé, sauf à la marge. Au fond, l'importance de la mutation envisagée explique très largement que les objectifs n'aient pu être réalisés. Pourtant, les lignes ont bougé durant ces années où elle s'est frottée à la question du multisupports. Pour ses dirigeants, les discours et les décisions qui ont abouti participent d'une question plus large, d'une vision entrepreneuriale à long terme : il s'agit de la question de la diversification.

Il y a une quinzaine d'années, le quotidien représentait la quasi-totalité du chiffre d'affaires du groupe. En 2010, sur les 135 millions d'euros d'activité annuelle $^{8}$, la part drainée par le titre n'en représente plus que les deux tiers ${ }^{9}$. Le PDG du groupe explique que désormais l'objectif est de ramener cette part à $50 \%$ à l'horizon 2015. Pour y parvenir, l'entreprise construit des marchés

\footnotetext{
8 « Le Télégramme, écosystème de la PQR », Stratégies, le 9 décembre 2010.

http://www.strategies.fr/actualites/medias/150472W/le-telegramme-ecosysteme-de-la-pqr.html

${ }^{9}$ Soit 88.890 .500 euros. Source : societe.com

http://www.societe.com/societe/s-a-le-telegramme-de-brest-et-de-l-ouest-925750325.html
} 
nouveaux, qui empêchent la concurrence de les exploiter à son profit et qui sont proches de son expertise : les médias, la communication. C'est en suivant cette logique entrepreneuriale que le groupe vient de prendre pied dans la télévision.

Cette stratégie de développement, on ne la comprend que si l'on intègre que l'entreprise fonctionne par tâtonnements, par essais-erreurs, par tentatives d'acculturation à une réalité mouvante en train de se faire. Pour ce qui nous concerne, on voit que ce qui a été appris avec le développement de l'internet et du multisupports est aujourd'hui recyclé dans cette chaîne de télévision. En 2007 a été essayé le JT-Web. Le public n'a pas suivi. Par contre, ce fut un vrai succès d'estime dans le monde des médias et au sein de la rédaction du titre. Les journalistes ont réalisé que leur entreprise était désormais dotée d'une expertise télévisuelle, un domaine jusqu'ici étranger et considéré comme une frontière de développement du groupe. Le JT-Web a constitué cette "étape préalable", qui avait surtout pour vocation de "faire entrer la vidéo dans l'univers papier du journal $\gg$.

L'essai va se concrétiser. Parce qu'en même temps qu'elle lance le JT-Web en 2007, l'entreprise se positionne sur ce qui n'est donc encore qu'un projet de télévision locale et régionale. Début 2009, le groupe obtient l'accord du CSA (Conseil supérieur de l'audiovisuel ${ }^{11}$ ) pour une fréquence locale TNT (Télévision numérique terrestre ${ }^{12}$ ). La chaîne Tébéo est partagée entre plusieurs actionnaires: deux communautés d'agglomération, une chambre consulaire $(5 \%)$, trois banques $(7,5 \%$ chacune), un club sportif $(3 \%)$, un autre titre de PQR (5\%), un club d'entreprises local (20\%), mais le groupe Télégramme est majoritaire $(51 \%)$. II installe ses studios dans les locaux de l'une de ses grandes rédactions, à Brest, et commence à émettre en novembre 2009. L'entreprise a engagé 1,5 million d'euros d'investissement de départ (création du studio dans la rédaction). La chaîne fonctionne avec un budget de 1,3 million d'euros. Pour le moment, elle perd de l'argent (250 000 euros après la première année), le but est d'atteindre l'équilibre économique fin $2014^{13}$. La maison mère assure le back-office de la chaîne, et facture ses prestations à la chaîne. Deux agents commerciaux de la régie publicitaire du groupe lui sont dédiés pour vendre de l'espace. La chaîne est une très petite structure, une équipe resserrée composée de quinze personnes, dont huit journalistes. Sept sont des transfuges de différents services de la rédaction, dont les anciens du JT-Web. Cette équipe

\footnotetext{
${ }^{10}$ Entretien réalisé auprès du rédacteur en chef multimédia.

${ }^{11}$ Le CSA est l'autorité française de régulation de la télévision et de la radio.

${ }^{12}$ La TNT, lancée en 2005 en France, est le standard de télédiffusion en France depuis novembre 2011.

${ }^{13}$ Conférence du rédacteur en chef multimédia, à l'IUT de Lannion, le 6 janvier 2011.
} 
Presse régionale et multisupports :

les petits pas de géants

produit près de deux heures de programmes quotidiens. Deux ans après son lancement, Tébéo est à la tête des audiences des chaînes locales (53.000 téléspectateurs/jour). Elle partage à peu près la zone de diffusion du journal papier, et l'entreprise souhaite, à terme, toucher la même audience (entre 600.000 et 800.000 personnes).

\section{Conclusion}

L'entreprise de presse que nous avons observée est coutumière de ce genre d'approche à petits pas. Une approche qui privilégie les procédures empiriques pour acquérir un savoir-faire pratique et pour construire progressivement ses choix stratégiques.

Au milieu des années 2000, la direction et la rédaction en chef ont écouté les discours omniprésents et souvent prophétiques sur les vertus du multisupports, elles ont côtoyé ses prosélytes, elles ont été attentives aux diverses tentatives de mise en œuvre d'un journalisme multisupports qui émergeaient ça et là. Mais sans plus. Elles n'ont jamais tout misé sur la mise en place d'un circuit de production de l'information qui serait totalement intégré, doté de journalistes capables de produire pour tous les supports.

Par contre, la manière dont elles se sont mêlées au journalisme multisupports leur a permis d'affirmer ce qu'elles sont, et ce qu'elles souhaitent devenir : une entreprise dont « le métier n'est plus de transporter des tonnes de papier sur les routes chaque nuit ${ }^{14}$, mais de transporter de l'information, via le papier, et aussi via son site internet ou via sa chaîne de télévision locale. L'entreprise est désormais en capacité à se définir comme un groupe en voie de diversification, capable de dépasser les frontières de son expertise historique. Un ensemble capable de se penser non plus comme une entreprise de presse, mais comme groupe industriel de communication.

\section{Bibliographie}

BOUQUILLION P., 2008, Les industries de la culture et de la communication. Les stratégies du capitalisme, Grenoble, PUG.

CABROLIÉ, S., 2009, « La recomposition d'une organisation de presse : le cas du Parisien.fr », Terrains \& travaux, vol. 1, $\mathrm{n}^{\circ} 15, \mathrm{pp} .127-145$.

CABROLIÉ, S., 2010, «Les journalistes du parisien.fr et le dispositif technique de production de l'information », Réseaux, vol.2, n³, pp. 79-100.

\footnotetext{
${ }^{14}$ Conférence du rédacteur en chef multimédia, à l'IUT de Lannion, le 6 janvier 2011.
} 
DEUZE M., 2005, « What is journalism? professional identity and ideology of journalists reconsidered ", Journalism, vol. 6, $\mathrm{n}^{\circ}$ 4, pp. 442-464.

DOMINGO D. et al., 2007, "Four dimensions of Journalistic Convergence : A Preliminary approach to current media trends in Spain ", dans VIII International Symposium of Online Journalism, 30 et 31 mars, University of Austin (Texas), [http://online.journalism.utexas.edu/2007/papers/Domingo.pdf], dernière consultation le 13 juin 2012.

FORTE DUHE, S., MORTIMER M-M., S-S. CHOW, 2004, "Convergence in North American TV Newsrooms : A Nationwide Look », Convergence : the international Journal of research into new media technologies, vol. 10, pp. 81-104.

GARCIA A., BIENVENIDO L., 2002, "Journalist Practice in Digital Television Newsrooms: the Case of Spain's Tele 5 and Antena 3 ", Journalism: Theory, Practice and Criticism, vol. 3, 2002, pp. 355-371.

GESTIN P. et al., 2009, " La production multisupports dans les groupes médiatiques français : premières remarques ", Les Cahiers du journalisme, $n^{\circ} 20$, automne, p. 84-95.

JENKINS H., 2004, "The Cultural Logic of Media Convergence », International Journal of Cultural Studies, vol. 7, n 1 , pp. 33-43.

QUINN, S., 2004, «An Intersection of Ideals: Journalism, Profits, Technology and Convergence ", Convergence : the international Journal of research into new media technologies, vol. 10, pp. 109-123.

QUINN, S., et QUINN-ALLAN, D., 2005, « The World-wide spread of journalism convergence ", Proceedings of the 2005 Journalism Education Association Conference, Griffith University (Australie), [http://dro.deakin.edu.au/eserv/DU: 30005866/quinn-worldwidespread-2005.pdf], dernière consultation le 13 juin 2012.

SINGER, J.B., 2004, «Strange Bedfellows? The diffusion of convergence in four news organizations ", Journalism Studies, vol. 5, $\mathrm{n}^{\circ}$ 1, pp. 3-18.

VOYENNE B., 1985, Les journalistes français : d'où viennent-ils ? qui sont-ils ? que font-ils ?, Paris, CFJ. 